\title{
Nutrient stress alters the glycosylation status of LGR5 resulting in reduced protein stability and membrane localisation in colorectal tumour cells: implications for targeting cancer stem cells
}

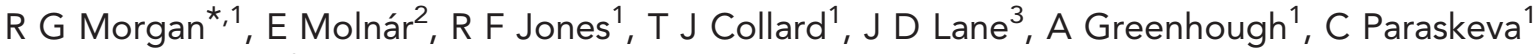 \\ and A C Williams ${ }^{1}$ \\ ${ }^{1}$ Cancer Research UK Colorectal Tumour Biology Group, School of Cellular and Molecular Medicine, Bristol BS8 1TD, UK; ${ }^{2}$ School \\ of Physiology and Pharmacology, Bristol BS8 1TD, UK and ${ }^{3}$ Cell Biology Laboratories, School of Biochemistry, University of Bristol, \\ Medical Sciences Building, University Walk, Bristol BS8 1TD, UK
}

Background: LGR5 is an important marker of intestinal stem cells and performs its vital functions at the cell membrane. Despite the importance of LGR5 to both normal and cancer stem cell biology, it is not known how microenvironmental stress affects the expression and subcellular distribution of the protein.

Methods: Nutrient stress was induced through glucose starvation. Glycosylation status was assessed using endoglycosidase or tunicamycin treatment. Flow cytometry and confocal microscopy were used to assess subcellular distribution of LGR5.

Results: Glucose deprivation altered the glycosylation status of LGR5 resulting in reduced protein stability and cell surface expression. Furthermore, inhibiting LGR5 glycosylation resulted in depleted surface expression and reduced localisation in the cisGolgi network.

Conclusions: Nutrient stress within a tumour microenvironment has the capacity to alter LGR5 protein stability and membrane localisation through modulation of LGR5 glycosylation status. As LGR5 surface localisation is required for enhanced Wnt signalling, this is the first report to show a mechanism by which the microenvironment could affect LGR5 function.

Leucine-rich repeat containing G-protein coupled receptor 5 (LGR5) is a seven-transmembrane protein recently identified as a marker of normal stem cells in several tissues including the intestine (Barker et al, 2013). LGR5 functions in the modulation of $\mathrm{Wnt} / \beta$-catenin signalling, a vital pathway in stem cell biology and tissue homeostasis. LGR5 binds extracellular R-spondins at the cell membrane (Carmon et al, 2011; de Lau et al, 2011) and subsequently associates with Wnt receptors to increase Wnt/ $\beta$-catenin signalling (Glinka et al, 2011; Carmon et al, 2012). Multiple studies have proposed that LGR5 identifies tumour-initiating cells, or promotes 'stemness' in colorectal cancer (CRC) (Barker et al, 2009; Kemper et al, 2012; Kobayashi et al, 2012; Schepers et al, 2012; Hirsch et al, 2013). Further studies suggest a pro-survival role in CRC cells, thus raising the potential of LGR5 as a therapeutic target in CRC (Al-Kharusi et al, 2013; Hirsch et al, 2013; Hsu et al, 2014). Nutrient deprivation occurs in a developing tumour when the metabolic demand exceeds that suppliable by the local vasculature (Vaupel et al, 1989). Such microenvironmental stresses can force molecular adaptations which promote a survival rather than proliferative response, resulting in clonal selection of a more malignant phenotype (Yun et al, 2009; Roberts et al, 2011). Despite 
the importance of LGR5 to stem cell biology, little is known about how expression and subcellular localisation are affected in microenvironmental pressures, such as nutrient stress. In this study, we show for the first time that LGR5 is glycosylated and that glucose deprivation alters the glycosylation status of LGR5 affecting the stability and subcellular localisation of the molecule.

\section{MATERIALS AND METHODS}

Cell lines and culture. The human colorectal adenoma-derived cell line RG/C2 was derived in this laboratory and maintained as described previously (Paraskeva et al, 1984, 1988; Williams et al, 1990). The LS174t-LGR5 cell line (double HA/FLAG tag) was a kind gift from Hans Clevers (de Lau et al, 2011). All other colorectal cell lines were obtained from the American Type Culture Collection and maintained as previously described (Moore et al, 2009).

Cell treatments. Deglycosylation of cell lysates by Endoglycosidase $\mathrm{H}$ (EndoH) and peptide $\mathrm{N}$-glycosidase $\mathrm{F}$ (PNGaseF) was performed as previously described (Molnar et al, 1995). For in vitro inhibition of glycosylation, cells were treated with $0.1-10 \mu \mathrm{g} \mathrm{ml}^{-1}$ tunicamycin (TMC; Sigma-Aldrich, Poole, UK) or the equivalent volume of DMSO vehicle control at $37^{\circ} \mathrm{C}$ for 24,48 and $72 \mathrm{~h}$. For inhibition of protein synthesis, cells were treated with $10 \mu \mathrm{g} \mathrm{ml}^{-1}$ cyclohexamide (Sigma), or the equivalent volume of water control, for $0-3 \mathrm{~h}$. Glucose starvation was performed as previously described (Roberts et al, 2011).

Immunoblotting. Immunoblotting was performed as described previously (Greenhough et al, 2010) using a previously validated rabbit monoclonal LGR5 antibody (ab75850, Abcam, Cambridge, UK) (Al-Kharusi et al, 2013).

Flow cytometry. For LGR5 surface staining, cells were immunolabelled with a previously validated primary rat anti-LGR5 antibody (4D11F8, Becton Dickinson, Oxford, UK) (Kemper et al, 2012) followed by secondary labelling with phycoerythrinconjugated goat anti-rat IgG (BD). For total LGR5 staining, cells were fixed in $2 \%$ paraformaldehyde and permeabilised using $0.1 \%$ Triton X-100 prior to antibody addition as above. Multi-parameter flow cytometric measurements were performed using FACSCanto II cytometer (BD) in conjunction with FACS Diva software v8.0 (BD). 7AAD (BD) was used to assess cell viability and the threshold for positive LGR5 staining was determined using the appropriately matched isotype control antibody (BD). Postacquisition analyses were performed using FlowJo software vX.0.6 (Tree Star Inc, Ashland, OR, USA).

Confocal laser scanning microscopy. Colorectal cancer cells were grown on glass coverslips for $72 \mathrm{~h}$ prior to DMSO control or TMC treatment. Treated cells were then stained for both total and surface LGR5 as above (see 'Flow Cytometry') except an Alexa488 goat anti-rat secondary antibody (Invitrogen, Paisley, UK) was preferred. Cells were co-stained with sheep anti-Grasp65 (Jon Lane, Bristol University) and Alexa647-conjugated anti-E-cadherin (cell membrane marker; BD) antibodies. Grasp65-stained cells were detected using an Alexa594-conjugated donkey antisheep secondary antibody (Invitrogen). All cells were counterstained with DAPI prior to mounting. Confocal laser scanning microscopy was performed as previously described (Petherick et al, 2013) and images were generated without any postacquisition manipulation using LAS AF v2.6.0 (Leica Microsystems, Milton Keynes, UK). Co-localisation quantification analyses were performed using Volocity confocal software v6.3.0 (Perkin-Elmer, Waltham, MA, USA).

Real-time polymerase chain reaction. Analysis of LGR5 mRNA was performed as previously described (Al-Kharusi et al, 2013).
Statistics. All statistical analyses were performed using GraphPad Prism v5.01 (GraphPad Software, Inc., San Diego, CA, USA). Significance of difference was assessed using one-sample or students $t$-test with significance defined at $P<0.05$.

\section{RESULTS}

To examine how tumour microenvironmental pressures such as nutrient stress affect LGR5 expression, we placed CRC cells into glucose-starved conditions. Following $16 \mathrm{~h}$ of glucose deprivation, both LoVo and LS174t-LGR5 cells not only demonstrated reduced expression of LGR5 but also exhibited a $10-20 \mathrm{kDa}$ lower molecular weight form of LGR5 (Figure 1A). Such protein mobility shifts can be the result of heavy posttranslational modifications such as glycosylation. Upon its initial characterisation, four consensus $N$-linked glycosylation sites were identified in the ectodomain of LGR5 (Hsu et al, 1998). Furthermore, using publicly available glycosylation site prediction software (NetNGlyc 1.0 Server, Technical University of Denmark, http:// www.cbs.dtu.dk/services/NetNGlyc (Blom et al, 2004)), we identified six potential glycosylation sites in the LGR5 amino acid sequence, three of which had strong confidence in the ectodomain. To formally explore the glycosylation status of LGR5, we treated cell lysate from LGR5-expressing colorectal tumour (CRT) cell lines with endoglycosidases that remove specific types of glycosylation. While EndoH removes immature forms only, PNGaseF removes all forms of $\mathrm{N}$-linked glycosylation. A panel of CRT cell lines were selected representing various stages of CRT progression including RG/C2 (adenoma), LS174t (carcinoma), SW620 and LoVo (advanced metastatic carcinoma). Treatment with either EndoH or PNGaseF resulted in a $10-20 \mathrm{kDa}$ molecular weight reduction in the LGR5 protein across all cell lines examined indicating LGR5 is glycosylated (Figure 1B). Across all CRT cell lines, there remained a readily detectable EndoH-resistant LGR5 protein at $99 \mathrm{kDa}$ confirming that both mature and immature glycosylated LGR5 protein are abundant within CRT cells. This is unsurprising given the very short half-life of LGR5 protein, which we estimated to be approximately $1 \mathrm{~h}$ using cyclohexamide treatment (Figure 1C). To examine the consequence of altered glycosylation status on expression and subcellular distribution of LGR5, it was important to be able to inhibit LGR5 glycosylation in living cells. To achieve this, we treated cells with an escalating dose of TMC, which broadly inhibits all $N$-linked glycosylation. As observed in Figure 1D, LGR5 glycosylation was optimally inhibited at $1 \mu \mathrm{g} \mathrm{ml}^{-1}$ TMC in most cell lines except for SW620 cells, which required a higher concentration of $5 \mu \mathrm{g} \mathrm{ml}^{-1}$. This is evidenced by the $10-20 \mathrm{kDa}$ loss in LGR5 molecular weight and reduced protein stability as previously observed with glucose deprivation (Figure 1D). We confirmed the above observations were likely due to a posttranslational modification of LGR5 by showing that LGR5 mRNA expression was not significantly affected by glucose starvation or TMC treatment (Figure 1E).

To examine the significance of LGR5 glycosylation status for its subcellular distribution within tumour cells, we treated CRT cell lines with TMC and assessed both total and cell surface LGR5 level by flow cytometry. TMC treatment significantly reduced total LGR5 levels in LoVo and LS174t-LGR5 cell lines by $67.7 \% \pm 12.4 \%$ and $45.6 \% \pm 15.2 \%$, respectively (Figure $2 \mathrm{~A}$ ). In comparison, cell surface LGR5 protein level was highly significantly diminished with TMC treatment in both cell lines. Disproportional to total LGR5 level, cell surface LGR5 was reduced by $98.7 \% \pm 1.9 \%$ and $90.8 \% \pm 6.6 \%$ for LoVo and LS174t-LGR5 cell lines, respectively (Figure 2B). To confirm these findings, we used confocal laser scanning microscopy which 
A

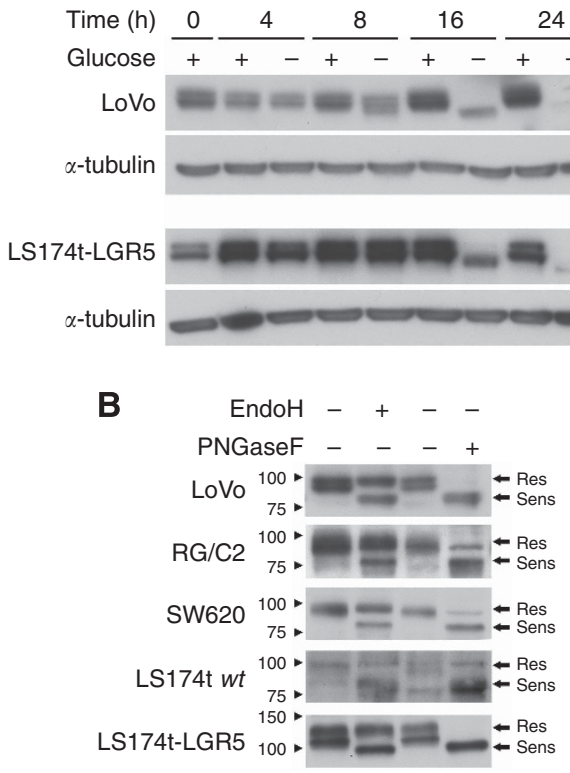

C

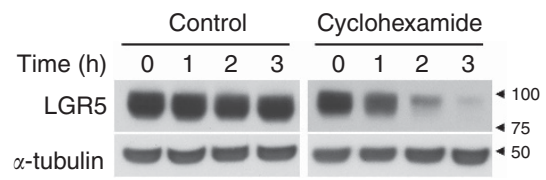

D $\operatorname{TMC}\left(\mu \mathrm{g} \mathrm{ml}^{-1}\right) \quad 0 \quad 0.1 \quad 0.5 \quad 1 \quad 5 \quad 10$

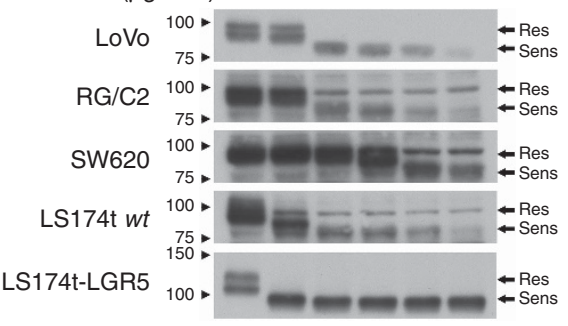

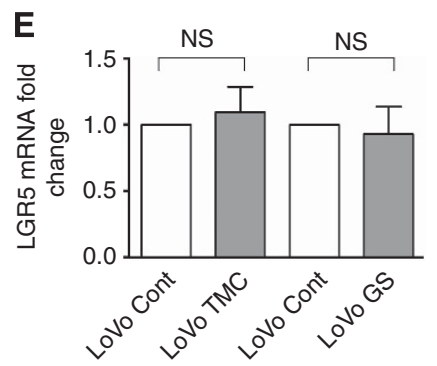

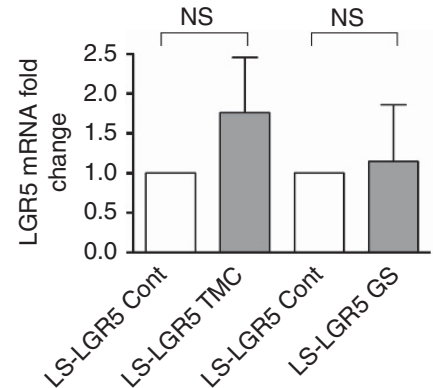

Figure 1. The glycosylation status of the LGR5 protein can be modified by glucose starvation and in vitro treatments. (A) Immunoblots showing LGR5 protein migration patterns from LoVo and LS174t-LGR5 cells cultured in glucose containing $(+)$ and non-glucose containing $(-)$ culture medium for 16 h. $\alpha$-Tubulin indicates comparable protein loading amongst samples whereas adjacent right-side arrowheads and numbers indicate position of molecular weight markers $(\mathrm{kDa}$ ) on blots. (B) Immunoblots showing LGR5 protein migration patterns from various CRT cell lines in response to endoglycosidase treatment or PBS control. Adjacent right-side arrows indicate the location of LGR5 forms either resistant (Res) or sensitive (Sens) to endoglycosidase treatment. Adjacent left-side arrowheads and numbers indicate position of molecular weight markers ( $\mathrm{kDa}$ ) on blots. Exposure times vary between all blots in order to clearly show LGR5 banding pattern and are thus not representative of relative LGR5 expression amongst the cell lines. Note the higher molecular weight of exogenous LGR5 protein in the LS174t-LGR5 line due to the presence of a double HA/FLAG tag. (C) Immunoblot showing LGR5 expression in LoVo cells treated with $10 \mu \mathrm{g} \mathrm{ml}^{-1}$ cyclohexamide or the equivalent water vehicle control for $0-3 \mathrm{~h}$. $\alpha$-Tubulin indicates comparable protein loading amongst samples. (D) Immunoblots showing LGR5 protein band conformation from CRT cell lines treated for $24 \mathrm{~h}$ with increasing doses of TMC. (E) Quantitative real-time PCR analysis of LGR5 mRNA in LoVo and LS174t-LGR5 cells following $16 \mathrm{~h}$ of glucose starvation (GS) or $24 \mathrm{~h} \mathrm{TMC}$ treatment. Data represent mean \pm 1 s.d., $n=3$. Statistical significance is denoted by ${ }^{\star \star} P<0.01,{ }^{\star \star *} P<0.001$ and $\mathrm{NS}=$ not significant as analysed by one-sample $t$-test.

demonstrated that LGR5 membrane expression was dramatically reduced in both TMC-treated LoVo and LS174t-LGR5 cells (Figure $2 \mathrm{C}$ and $\mathrm{D}$, respectively). Detection of E-cadherin in LoVo cells served not only as a membrane marker, but also demonstrated that TMC treatment does not universally deplete cell surface expression of all glycosylated membrane proteins such as E-cadherin (Figure 2E) (Zhao et al, 2008). Detection of E-cadherin in the LS174t cell line was not possible in keeping with a previously reported $\mathrm{CDH} 1$ mutation (Efstathiou et al, 1999). To confirm that TMC treatment affected LGR5 in a similar manner to nutrient stress, we repeated our flow cytometry experiments in Figure 2A but with glucose starvation and also observed significantly depleted LGR5 surface expression (Figure 2F). We also confirmed that 16-h glucose deprivation did not alter cell viability (Figure $2 \mathrm{G}$ ).

Following initial glycosylation events in the endoplasmic reticulum, newly synthesised proteins are transported to the cisGolgi network where further glycosylation trafficks the protein to the appropriate subcellular compartment. Using Grasp65 as a marker of the cis-Golgi network (Cheng et al, 2010), we examined LGR5 localisation after inhibition of glycosylation. For both DMSO control-treated LoVo and LS174t-LGR5 cells, intracellular LGR5 staining presented mainly as large intracellular puncta within the cytoplasm and perinuclear regions (Figure $3 \mathrm{~A}$ and B, respectively) as previously reported (Kemper et al, 2012; Snyder et al, 2013). A substantial proportion of the LGR5 signal co-localised with Grasp65 as indicated by areas of white co-fluorescence. In TMC-treated LoVo cells, the overall LGR5 signal was clearly reduced as previously observed in flow cytometry and immunoblot experiments, again suggesting reduced overall protein stability. However, the remaining LGR5 signal appeared more dispersed within the cytoplasm with several Grasp65-positive regions devoid of any LGR5 co-staining (Figure 3A and B). Co-localisation quantification analyses using both Pearson's Correlation Coefficient and Mander's Colocalisation Coefficient (Manders et al, 1993) confirmed these observations. For both LoVo and LS174t-LGR5 cells, there existed a strong positive correlation between LGR5 and Grasp65 signal which was 
A

$$
\begin{aligned}
\text { A.... } & =\text { Autofluor. } \\
-\cdot-\cdot- & =\text { Isotype } \\
& =\text { LGR5 (Cont) } \\
& =\text { LGR5 (TMC) }
\end{aligned}
$$

LoVo

LS174t-LGR5

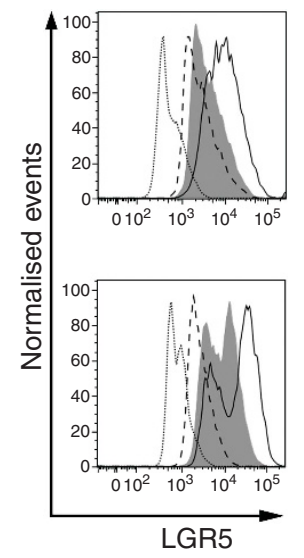

C

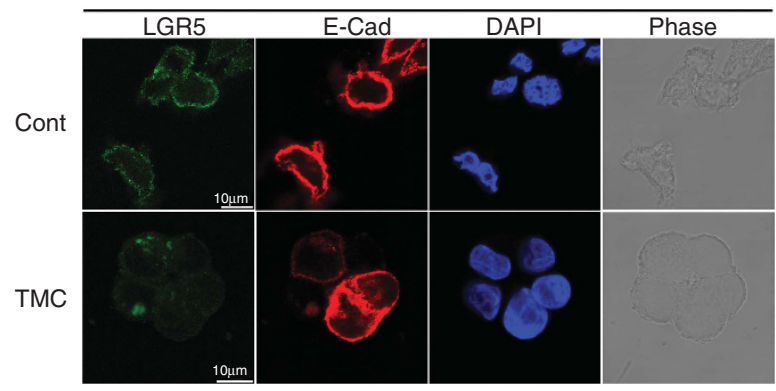

E
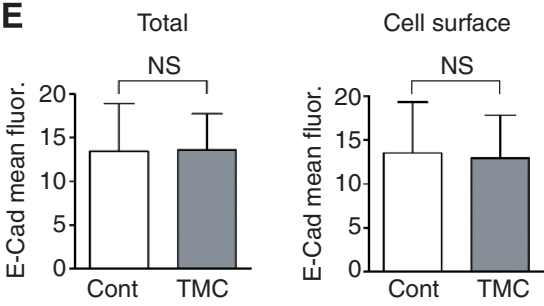

Total
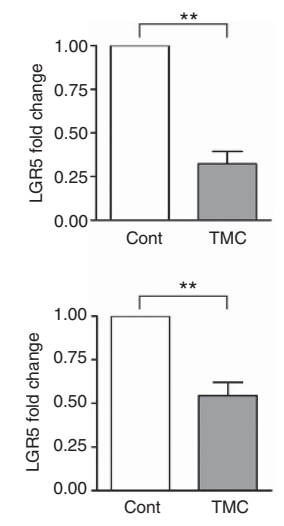

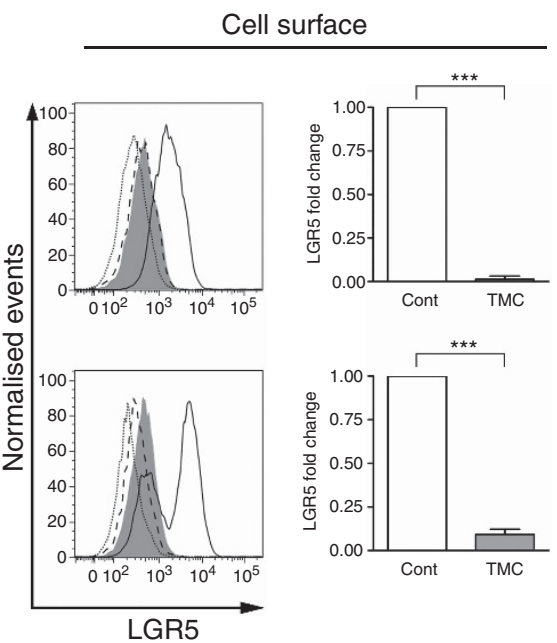

D

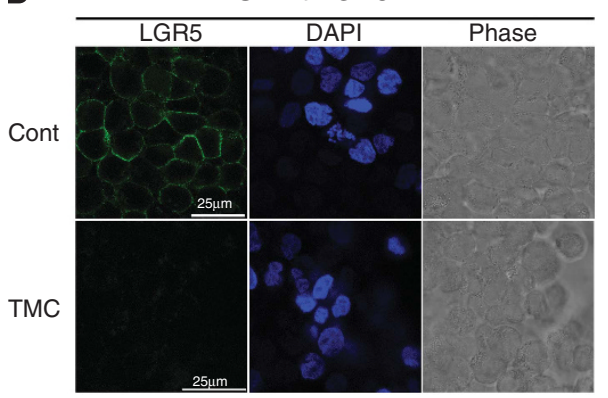

$\mathbf{F}$

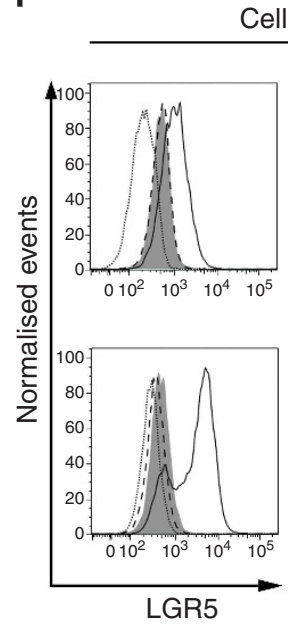

Cell surface
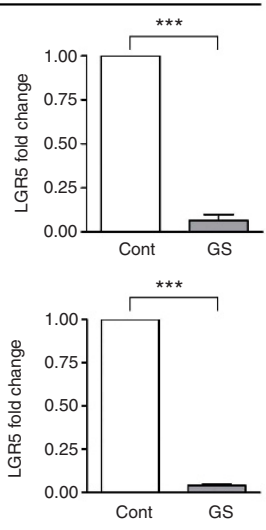

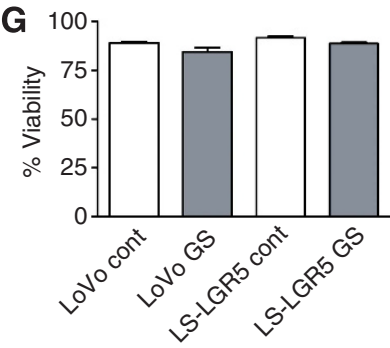

LS174t-LGR5

\section{LoVo}

Figure 2. Inhibiting glycosylation of LGR5 results in severely depleted cell surface expression. Representative flow cytometric histograms and associated summary graphs demonstrating the (A) total or (B) cell surface expression of LGR5 in response to $24 \mathrm{~h}$ DMSO control (Cont) or $1 \mu \mathrm{g} \mathrm{ml}^{-1} \mathrm{TMC}$ treatment. Representative confocal laser scanning microscopy Z-sections showing LGR5 cell surface expression in (C) LoVo and (D) LS174t-LGR5 cells following $24 \mathrm{~h}$ DMSO control (Cont) or $1 \mu \mathrm{g} \mathrm{ml}^{-1} \mathrm{TMC}$ treatment. LGR5 (green), E-cadherin (E-Cad; red), DAPI (blue) and Phase (grey) images are shown. Data shown are representative of 20 fields each derived from three independent experiments. (E) Quantification of total and cell surface E-cadherin mean fluorescence from control and TMC treated LoVo cells as analysed by confocal laser scanning microscopy. (F) Representative flow cytometric histograms and associated summary graphs showing the cell surface expression of LGR5 in LoVo and LS174t-LGR5 cells following $16 \mathrm{~h}$ glucose starvation (GS). (G) Flow cytometric assessment of percentage viability in control and glucose-starved cultures ( $>80 \%$ as deduced from 7AAD dye). For all graphs, data represent mean \pm 1 s.d., $n=3$. Statistical significance is denoted by ${ }^{\star \star} P<0.01,{ }^{\star \star \star} P<0.001$ and NS = not significant as analysed by one-sample t-test.

significantly weakened upon TMC treatment in both cell lines (Figure 3C and D, respectively). These data indicate that the absence of $\mathrm{N}$-linked glycosylation results in a lower proportion of
LGR5 localised within the cis-Golgi network, consistent with the notion that incorrectly folded proteins are not trafficked to the Golgi. 
A

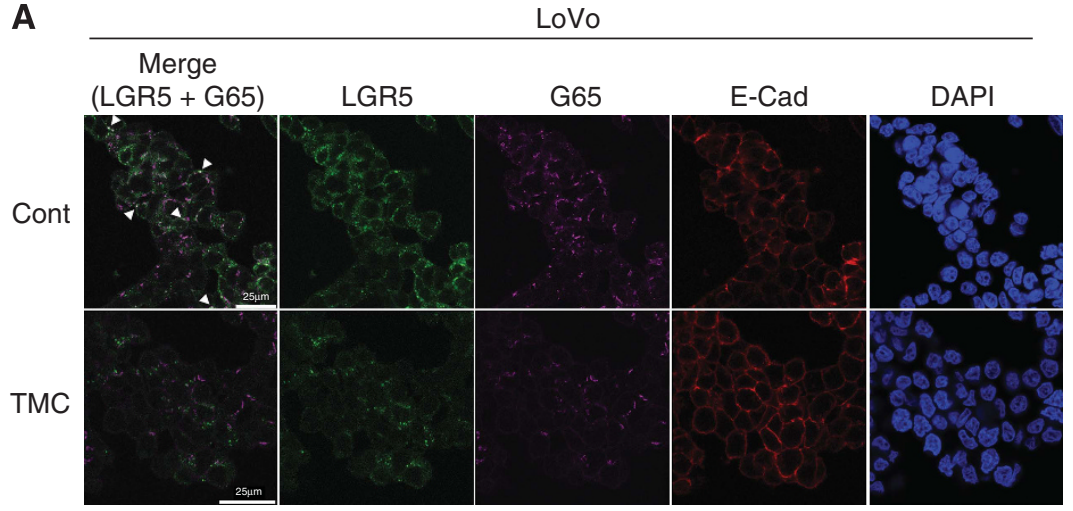

$\mathbf{B}$

LS174t-LGR5

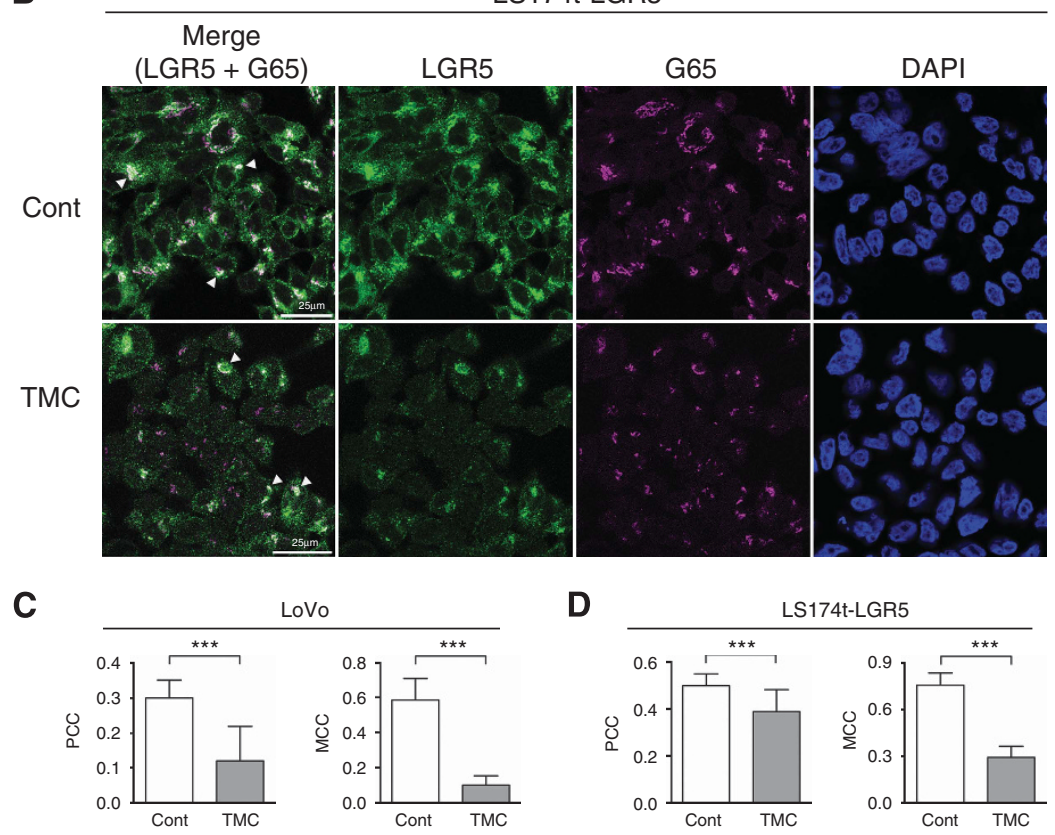

Figure 3. Inhibiting LGR5 glycosylation results in reduced localisation within the cis-Golgi network. Confocal laser scanning microscopy Z-sections showing intracellular LGR5 expression in fixed and permeabilised (A) LoVo and (B) LS174t-LGR5 cells following $24 \mathrm{~h}$ treatment with DMSO control or $1 \mu \mathrm{g} \mathrm{ml}^{-1}$ TMC. Merged (LGR5 and Grasp65 only), LGR5 (green), Grasp65 (G65; magenta), E-cadherin (E-Cad; red) and DAPI (blue) images are shown. Areas of co-localisation between LGR5 and Grasp65 are indicated by resulting white colour (highlighted by arrowheads). Overall correlation between LGR5 and Grasp65 signal in DMSO control or TMC treated (C) LoVo and (D) LS174t-LGR5 cells, as determined by thresholded Pearsons Correlation Coefficient $(-1=$ inverse correlation, $0=$ no correlation, $+1=$ positive correlation). Proportion of Grasp65 signal co-localised with LGR5 fluorescence in DMSO control or TMC treated cells is also shown as determined by the Mander's Colocalisation Coefficient. Data represent mean $\pm 1 \mathrm{~s}$.d. as deduced from a minimum of 20 random fields, $n=3$. Statistical significance is denoted by ${ }^{\star \star \star} P<0.001$, as analysed by students $t$-test.

\section{DISCUSSION}

This study shows for the first time that LGR5 is glycosylated and importantly that nutrient stress can alter the glycosylation status of LGR5 resulting in reduced protein stability and membrane localisation in CRT cells. The main LGR5 stem cell function described to date is in the regulation of Wnt signalling through capturing of R-Spondins and complexing with the Wnt receptors Frz and LRP5/6 (Carmon et al, 2011; de Lau et al, 2011). Therefore, glycosylation represents a critical posttranslational modification allowing LGR5 to perform these, and other potential uncharacterised functions at the membrane. It is conceivable that a tumour-initiating cell, within a glucose-deprived microenvironment, could obtain a competitive advantage through reduced LGR5 surface levels, by directing the cell into a pro-survival mode rather than the proliferative response associated with Wnt signalling promotion. This study also has implications for targeting cancer stem cells through surface markers, such as LGR5, because surface expression is significantly reduced by fluctuating tumour microenvironmental pressures such as nutrient stress.

\section{ACKNOWLEDGEMENTS}

Thanks to Dr. Andrew Herman and the University of Bristol flow cytometry suite for assistance with flow cytometric assays. Thanks to the Wolfson Bioimaging Facility (University of Bristol) for aiding confocal microscopy experiments. Finally, we thank Dr. Anthony Corfield for critical appraisal of the manuscript. This work was supported by a Cancer Research UK Programme Grant (C19/A11975). EM's research is supported by the Biotechnology and Biological Sciences Research Council, UK (BB/J015938/1). 


\section{CONFLICT OF INTEREST}

No conflict of interest declared.

\section{AUTHOR CONTRIBUTIONS}

RGM designed and executed experiments and wrote the manuscript. RFJ and TJC performed experiments and analysed data. JDL provided reagents and experimental guidance. AG, ACW, EM and $\mathrm{CP}$ gave project direction and co-wrote the manuscript.

\section{REFERENCES}

Al-Kharusi MR, Smartt HJ, Greenhough A, Collard TJ, Emery ED, Williams AC, Paraskeva C (2013) LGR5 promotes survival in human colorectal adenoma cells and is upregulated by PGE2: implications for targeting adenoma stem cells with NSAIDs. Carcinogenesis 34(5): 1150-1157.

Barker N, Ridgway RA, van Es JH, van de Wetering M, Begthel $H$, van den Born M, Danenberg E, Clarke AR, Sansom OJ, Clevers H (2009) Crypt stem cells as the cells-of-origin of intestinal cancer. Nature 457(7229): 608-U119.

Barker N, Tan S, Clevers H (2013) Lgr proteins in epithelial stem cell biology. Development 140(12): 2484-2494.

Blom N, Sicheritz-Ponten T, Gupta R, Gammeltoft S, Brunak S (2004) Prediction of post-translational glycosylation and phosphorylation of proteins from the amino acid sequence. Proteomics 4(6): 1633-1649.

Carmon KS, Gong X, Lin Q, Thomas A, Liu Q (2011) R-spondins function as ligands of the orphan receptors LGR4 and LGR5 to regulate Wnt/ beta-catenin signaling. Proc Natl Acad Sci USA 108(28): 11452-11457.

Carmon KS, Lin Q, Gong X, Thomas A, Liu Q (2012) LGR5 interacts and cointernalizes with Wnt receptors to modulate Wnt/beta-catenin signaling. Mol Cell Biol 32(11): 2054-2064.

Cheng JP, Betin VM, Weir H, Shelmani GM, Moss DK, Lane JD (2010) Caspase cleavage of the Golgi stacking factor GRASP65 is required for Fas/CD95-mediated apoptosis. Cell Death Dis 1: e82.

de Lau W, Barker N, Low TY, Koo BK, Li VS, Teunissen H, Kujala P, Haegebarth A, Peters PJ, van de Wetering M, Stange DE, van Es JE, Guardavaccaro D, Schasfoort RB, Mohri Y, Nishimori K, Mohammed S, Heck AJ, Clevers H (2011) Lgr5 homologues associate with Wnt receptors and mediate R-spondin signalling. Nature 476(7360): 293-297.

Efstathiou JA, Liu D, Wheeler JM, Kim HC, Beck NE, Ilyas M, Karayiannakis AJ, Mortensen NJ, Kmiot W, Playford RJ, Pignatelli M, Bodmer WF (1999) Mutated epithelial cadherin is associated with increased tumorigenicity and loss of adhesion and of responsiveness to the motogenic trefoil factor 2 in colon carcinoma cells. Proc Natl Acad Sci USA 96(5): 2316-2321.

Glinka A, Dolde C, Kirsch N, Huang YL, Kazanskaya O, Ingelfinger D, Boutros M, Cruciat CM, Niehrs C (2011) LGR4 and LGR5 are R-spondin receptors mediating Wnt/beta-catenin and Wnt/PCP signalling. EMBO Rep 12(10): 1055-1061.

Greenhough A, Wallam CA, Hicks DJ, Moorghen M, Williams AC, Paraskeva C (2010) The proapoptotic $\mathrm{BH} 3$-only protein Bim is downregulated in a subset of colorectal cancers and is repressed by antiapoptotic COX-2/ PGE(2) signalling in colorectal adenoma cells. Oncogene 29(23): 3398-3410.

Hirsch D, Barker N, McNeil N, Hu Y, Camps J, McKinnon K, Clevers H, Ried T, Gaiser T (2013) LGR5 positivity defines stem-like cells in colorectal cancer. Carcinogenesis 35(4): 849-858.

Hsu HC, Liu YS, Tseng KC, Tan BC, Chen SJ, Chen HC (2014) LGR5 regulates survival through mitochondria-mediated apoptosis and by targeting the Wnt/beta-catenin signaling pathway in colorectal cancer cells. Cell Signal 26(11): 2333-2342.

Hsu SY, Liang SG, Hsueh AJ (1998) Characterization of two LGR genes homologous to gonadotropin and thyrotropin receptors with extracellular leucine-rich repeats and a $\mathrm{G}$ protein-coupled, seven-transmembrane region. Mol Endocrinol 12(12): 1830-1845.

Kemper K, Prasetyanti PR, De Lau W, Rodermond H, Clevers H, Medema JP (2012) Monoclonal antibodies against Lgr5 identify human colorectal cancer stem cells. Stem Cells 30(11): 2378-2386.

Kobayashi S, Yamada-Okabe H, Suzuki M, Natori O, Kato A, Matsubara K, Jau Chen Y, Yamazaki M, Funahashi S, Yoshida K, Hashimoto E, Watanabe Y, Mutoh H, Ashihara M, Kato C, Watanabe T, Yoshikubo T, Tamaoki N, Ochiya T, Kuroda M, Levine AJ, Yamazaki T (2012) LGR5positive colon cancer stem cells interconvert with drug-resistant LGR5negative cells and are capable of tumor reconstitution. Stem Cells 30(12): 2631-2644.

Manders EMM, Verbeek FJ, Aten JA (1993) Measurement of Colocalization of Objects in Dual-Color Confocal Images. J Microsc-Oxford 169: 375-382.

Molnar E, Varadi A, McIlhinney RA, Ashcroft SJ (1995) Identification of functional ionotropic glutamate receptor proteins in pancreatic beta-cells and in islets of Langerhans. FEBS Lett 371(3): 253-257.

Moore AE, Greenhough A, Roberts HR, Hicks DJ, Patsos HA, Williams AC, Paraskeva C (2009) HGF/Met signalling promotes PGE(2) biogenesis via regulation of COX-2 and 15-PGDH expression in colorectal cancer cells. Carcinogenesis 30(10): 1796-1804.

Paraskeva C, Buckle BG, Sheer D, Wigley CB (1984) The isolation and characterization of colorectal epithelial cell lines at different stages in malignant transformation from familial polyposis coli patients. Int $J$ Cancer 34(1): 49-56.

Paraskeva C, Finerty S, Powell S (1988) Immortalization of a human colorectal adenoma cell line by continuous in vitro passage: possible involvement of chromosome 1 in tumour progression. Int J Cancer 41(6): 908-912.

Petherick KJ, Williams AC, Lane JD, Ordonez-Moran P, Huelsken J, Collard TJ, Smartt HJ, Batson J, Malik K, Paraskeva C, Greenhough A (2013) Autolysosomal beta-catenin degradation regulates Wnt-autophagy-p62 crosstalk. EMBO J 32(13): 1903-1916.

Roberts HR, Smartt HJ, Greenhough A, Moore AE, Williams AC, Paraskeva C (2011) Colon tumour cells increase PGE(2) by regulating COX-2 and 15PGDH to promote survival during the microenvironmental stress of glucose deprivation. Carcinogenesis 32(11): 1741-1747.

Schepers AG, Snippert HJ, Stange DE, van den Born M, van Es JH, van de Wetering M, Clevers $\mathrm{H}$ (2012) Lineage tracing reveals Lgr5 + stem cell activity in mouse intestinal adenomas. Science 337(6095): 730-735.

Snyder JC, Rochelle LK, Lyerly HK, Caron MG, Barak LS (2013) Constitutive internalization of the leucine-rich G protein-coupled receptor-5 (LGR5) to the trans-Golgi network. J Biol Chem 288(15): 10286-10297.

Vaupel P, Kallinowski F, Okunieff P (1989) Blood flow, oxygen and nutrient supply, and metabolic microenvironment of human tumors: a review. Cancer Res 49(23): 6449-6465.

Williams AC, Harper SJ, Paraskeva C (1990) Neoplastic transformation of a human colonic epithelial cell line: in vitro evidence for the adenoma to carcinoma sequence. Cancer Res 50(15): 4724-4730.

Yun J, Rago C, Cheong I, Pagliarini R, Angenendt P, Rajagopalan H, Schmidt K, Willson JK, Markowitz S, Zhou S, Diaz Jr. LA, Velculescu VE, Lengauer C, Kinzler KW, Vogelstein B, Papadopoulos N (2009) Glucose deprivation contributes to the development of KRAS pathway mutations in tumor cells. Science 325(5947): 1555-1559.

Zhao H, Liang Y, Xu Z, Wang L, Zhou F, Li Z, Jin J, Yang Y, Fang Z, Hu Y, Zhang L, Su J, Zha X (2008) N-glycosylation affects the adhesive function of E-Cadherin through modifying the composition of adherens junctions (AJs) in human breast carcinoma cell line MDA-MB-435. J Cell Biochem 104(1): 162-175. 